\title{
Divulgación del conocimiento en el ciberespacio: un viaje a través del multilingüismo
}

\author{
Popularizing knowledge in the cyberspace: \\ a travel through multilingualism
}

Mercedes Sanz Gil UNIVERSITAT JAUME I

Recibido: $15 / 02 / 2014$

Aceptado: 23/05/2014

ABSTRACT: The universal declaration of the UNESCO on the cultural diversity of 2001 explicitly show, among others, the right of the citizens to express, create and spread their works in the language of their choice, the right to an education and a quality formation that respect completely their cultural identity and the right to take part of the cultural life of their choice. In this paper we present some multilingual initiatives in the cyberspace that fight day after day for the respect and the defense of the fundamental rights of the languages and the cultures, despite its minority use, opposite to the linguistic imperialism, promoting in addition, the communication of the science and the research through them.

Keywords: cyberspace, plurilingualism, cultural diversity, spreading of the knowledge.

RESUMEN: La declaración universal de la UNESCO sobre la diversidad cultural de 2001 manifiesta explícitamente, entre otros, el derecho de los ciudadanos a expresarse, crear y difundir sus obras en la lengua de su elección, el derecho a una educación y una formación de calidad que respeten plenamente su identidad cultural y el derecho a participar de la vida cultural de su elección. En este trabajo se presentan algunas iniciativas multilingües presentes en el ciberespacio que luchan día a día por el respeto y la defensa de los derechos fundamentales de las lenguas y de las culturas, por minoritarias que sean, frente al imperialismo lingüístico, promoviendo además, la comunicación de la ciencia y de la investigación a través de ellas.

Palabras clave: ciberespacio, plurilingüismo, diversidad cultural, divulgación del conocimiento. 
La beauté d'un tapis réside dans la variété de ses couleurs.

S'il n'y a que du blanc, ce serait un drap blanc; s'il n'y a que du noir, ce serait un pagne de deuil. C'est l'Univers tout entier qui est notre patrie.

Chacun de nous est une page du grand livre de la Nature. Dans la vaste communauté humaine lancée à la recherche d'un nouvel équilibre, chaque peuple doit apporter la note de son génie propre afin que tout l'ensemble en soit enrichi.

Chacun doit s'ouvrir aux autres tout en restant lui-même. ${ }^{1}$

AmAdou HampÂTÉ

\section{Introducción}

La cita del escritor y filósofo malí Amadou Hampâté con la que abrimos este artículo resume claramente la esencia de la diversidad cultural y lingüística.

Una muestra de esta variedad de colores que hacen bello un tapiz la tenemos en la misma Unión Europea que cuenta en la actualidad con $24^{2}$ lenguas reconocidas oficialmente, además de las más de 60 lenguas autóctonas minoritarias o regionales y un buen número también de lenguas no autóctonas habladas por las diferentes comunidades de emigrantes.

Es la aceptación de esta realidad plural, de esta variedad de colores, la que da a cada individuo, a cada comunidad, a cada sociedad sus derechos fundamentales, tal y como lo estipula también la declaración de la UNESCO sobre la diversidad cultural, en su artículo 5 (2001):

Toda persona debe, así, poder expresarse, crear y difundir sus obras en la lengua que desee y en particular en su lengua materna; toda persona tiene derecho a una educación y una formación de calidad que respete plenamente su identidad cultural; toda persona debe poder participar en la vida cultural que elija y ejercer sus propias prácticas culturales, dentro de los límites que impone el respeto de los derechos humanos y de las libertades fundamentales.

1. En Adama Masséekou (2012): «L'avenir se parle, s'écrit et se lit dans toutes les langues», NET. LANG. Réussir dans le cyberespace multilingue, Caen, C\&F Éditions.

2. Alemán, búlgaro, checo, croata, danés, eslovaco, esloveno, español, estonio, finés, francés, griego, húngaro, inglés, irlandés, italiano, letón, lituano, maltés, neerlandés, polaco, portugués, rumano y sueco. 
La Unión Europea se ha comprometido a preservar esta diversidad lingüística y a promover el conocimiento de las lenguas en nombre de la identidad cultural, de la integración y de la cohesión social.

Tras los resultados del último Eurobarómetro de 2012 sobre «Los europeos y sus lenguas» que expondremos más adelante, la Comisión Europea quiere, además, incrementar el apoyo al aprendizaje de lenguas gracias al nuevo programa 2014-2020 Erasmus para todos, materializado en el programa Erasmus.$+{ }^{3}$ Dicho programa pretende favorecer la movilidad de estudiantes y trabajadores a nivel internacional, no solo en territorio europeo, englobando todas las iniciativas de educación, formación, juventud y deporte, y abarcando en materia educativa a todos los niveles formativos: escolar, formación profesional, enseñanza superior y formación de personas adultas.

La influencia de la Comisión Europea es, sin embargo, limitada en el ámbito de la educación y de las políticas lingüísticas, ya que estas son competencia particular de los estados miembros y en ocasiones llegan a producirse contradicciones entre las recomendaciones de los expertos en materia de lenguas, la Dirección General de Educación y Cultura de la Unión Europea, y la interpretación y puesta en práctica de las políticas estatales. Si bien la primera considera prioritario establecer un plan estratégico a favor del multilingüismo, las segundas siguen desarrollando políticas más encaminadas a impulsar el aprendizaje de una única lengua.

Preservar y promover la diversidad y el pluralismo culturales y lingüísticos en cualquier espacio resulta, desde este punto de vista, una necesidad imperiosa.

El ciberespacio, cada vez más cotidiano para los usuarios adultos, es por otra parte el entorno natural de comunicación y de aprendizaje de las generaciones que hoy se encuentran en edad escolar y que en un futuro cercano tendrán que defender estos derechos fundamentales.

Como no podía ser de otra manera, contrariamente a lo que pueden pensar los defensores de una lengua única, de una lengua común para todos, de una

3. Programa aprobado por el Parlamento Europeo para el periodo 2014-2020 y en vigor desde el 1 de enero de 2014. Este programa se enmarca en la estrategia Europa 2020, en la estrategia Educación y Formación 2020 y en la estrategia Rethinking Education y engloba todas las iniciativas de educación, formación, juventud y deporte. En materia educativa abarca todos los niveles: escolar, formación profesional, enseñanza superior y formación de personas adultas, e integrará los programas existentes en el Programa de Aprendizaje Permanente y también los programas de educación superior internacional: Mundus, Tempus, ALFA, Edulink y programas bilaterales, además del Programa Juventud en Acción. 
lingua franca en la era de la globalización, este ciberespacio es un entorno, al igual que la naturaleza, teñido y plagado de color cultural y lingüístico. En la sociedad actual, este ciberespacio juega un papel esencial como fuente difusora del multilingüismo real de las sociedades y por ende como espacio mediador para la divulgación del saber. El ciberespacio constituye un medio de información, pero también de comunicación a través de las tecnologías sociales (blogs, wikis, redes sociales) entre usuarios favoreciendo tanto la transmisión de conocimientos como la interacción, lo que la convierte en un espacio privilegiado para la divulgación, la discusión, el debate, la argumentación, es decir, las diferentes operaciones implicadas en el desarrollo del saber.

En este trabajo damos cuenta de esta realidad presentando algunas iniciativas multilingües del ciberespacio que luchan día a día por el respeto y la defensa de los derechos fundamentales de las lenguas y de las culturas, por minoritarias que sean, frente al imperialismo lingüístico, promoviendo, además, la comunicación y divulgación de la ciencia y de la investigación a través de ellas con el fin de reducir la brecha informacional provocada por el unilingüismo, que afecta a una buena parte de la población del planeta.

\section{Mito y realidad de una lingua franca ${ }^{4}$}

El sentido original del término lingua franca se refiere a la lengua hablada hasta el siglo XIX en los puertos mediterráneos con fines comerciales principalmente; era una lengua compuesta a base de términos del castellano, catalán, francés, provenzal, italiano, genovés y veneciano mezclada con elementos del griego y del árabe (Fondevila y Sánchez, 2004-2007: 157). Se trataba de una lengua puramente utilitaria, un híbrido compuesto por varias lenguas. Por el contrario, este término se refiere hoy en día, por extensión, a otra realidad: se trataría más bien de una lengua vehicular utilizada en un área geográfica bastante amplia entre hablantes de diferente lengua materna. Este es el sentido que recoge el Diccionario de la Real Academia de la Lengua Española definiéndola como «lengua que es mezcla de dos o más, y con la cual se entienden los naturales de pueblos distintos»».

Una idea muy extendida hasta hace poco es que el inglés sería la lingua franca en la era de la globalización, lo que le valió en algunos sectores el calificativo de «lengua universal»(Global English). En este contexto el inglés

4. Título tomado parcialmente de una sección del coloquio internacional Communication et mondialisation. Les limites du tout-anglais, CNRS, París, 14/11/2012. 
sería la única lengua que necesitaríamos para comunicar, por su sencillez y por permitir economizar recursos, ya que no habría necesidad de traducir a ninguna lengua. Ahora bien, la realidad actual es bien diferente incluso en el seno del mundo anglófono, en el que el modelo del «todo inglés» se está cuestionando tanto en el Reino Unido como en los Estados Unidos de América.

En efecto, un informe de $2009^{5}$ de la prestigiosa British Academy for the Humanities and Social Sciences alerta sobre la poca formación en lenguas modernas que tienen sus investigadores provocando que se encuentren en clara desventaja para competir con sus homólogos en el extranjero, que se reduzca la capacidad del Reino Unido para abordar muchos desafíos mundiales, que afecte a la economía del país y que dañe la salud de las humanidades y de las ciencias sociales.

Por otra parte, en la Conferencia de Rectores de Universidad de Alemania del 22-11-2011 en la que se abordó este tema, se llegó a la conclusión de que «la imposición creciente de la utilización del inglés en la investigación puede perjudicar seriamente la calidad y la eficacia del trabajo de los investigadores, del mismo modo que la falta de consideración de otras lenguas en las publicaciones distorsiona el concepto de la competencia».

En este encuentro manifestaron igualmente la necesidad de poner en marcha políticas que doten a los departamentos universitarios de los efectivos necesarios para la enseñanza e investigación en lenguas, así como de servicios suplementarios como traducción, corrección e interpretación. Mencionaron igualmente la financiación de sistemas bibliométricos europeos destinados a las publicaciones en otras lenguas diferentes del inglés de manera que reciban la atención que merecen.

Después de una tendencia generalizada a favorecer la investigación en inglés, en aras de conseguir una lengua de la ciencia, una lingua franca, ahora las instituciones alemanas han vuelto a considerar, promocionar y favorecer que sus doctorandos realicen sus investigaciones y sus tesis doctorales en otras lenguas diferentes del inglés, en la propia por supuesto, pero en cualquier otra también.

Otro caso también, ahora fuera de Europa, es el de Canadá, que no solo está empezando a permitir sino también a promocionar la defensa de tesis doctorales en lenguas autóctonas con traducción.

En paralelo al fenómeno de la globalización existe otro fenómeno cada vez más creciente que es el de la multipolaridad y desoccidentalización, con nuevos países que se están creando, países y culturas emergentes política y

5. Informe del 3 de junio de 2009: http://www.britac.ac.uk/policy/language-matters.cfm 
económicamente hablando, basta con mencionar los ejemplos de los países del Este de Europa, Sudamérica o África (Brasil, Rusia, India, China: BRIC). Hay empresas que se están haciendo eco de ello, como el New York Times que desde 2013 publica una edición en portugués destinado al público brasileño, que se convierte poco a poco en potencia comercial. Y es que, en términos de Michaël Oustinoff (2012), «las pequeñas lenguas de hoy pueden ser las grandes lenguas de mañana», cuestionando así la validez del predominio de una lingua franca y de su rentabilidad económica, argumento esgrimido habitualmente en su favor.

En efecto, este planteamiento de Oustinoff nos lleva a repensar la rentabilidad de esa lingua franca. A priori, si pensamos en términos estrictamente económicos y desde una perspectiva de simplificación, se puede afirmar que es rentable contar con una lingua franca, puesto que si todos hablamos una única lengua, las administraciones ahorrarían los costes de traducción; sin embargo, como dice François Grin (2012), el ahorro en traducción es un gasto en formación en una única lengua (lengua dominante) a los no nativos. Por ejemplo, en la Unión Europea habría que formar a los 500 millones de habitantes para que pudieran comunicarse entre ellos, y no hay que olvidar que son hablantes de 24 lenguas reconocidas. ${ }^{6}$

Esta tarea se presenta mucho más complicada todavía si se tienen en cuenta los datos del último Eurobarómetro de 2012 sobre los europeos y sus lenguas, en el que los resultados revelan que la mayoría de los europeos no se describen a sí mismos como aprendedores activos de idiomas: en torno a una cuarta parte (un $23 \%$ ) de los europeos nunca ha estudiado un idioma, mientras que algo más de dos quintas partes (un $44 \%$ ) no lo han hecho recientemente ni tiene intención de empezar; solo una minoría (un $14 \%$ ) ha seguido aprendiendo un idioma en los últimos dos años; menos de uno de cada diez (un $7 \%$ ) ha empezado a aprender un idioma en los últimos dos años y una proporción similar (un $8 \%$ ) no ha estudiado un idioma recientemente, pero tiene intención de empezar al año siguiente. No obstante, prácticamente la totalidad de los encuestados (98\%) declara que es bueno que sus hijos aprendan idiomas.

Si tenemos en cuenta que un estudio realizado recientemente en la Unión Europea ha revelado que el coste en traducción es de 2,20 € por habitante y año (Grin, 2012), el término de rentabilidad económica asociado a una lingua franca evidentemente no se revela como el más acertado. En el mismo sentido

6. Intervención realizada en la mesa redonda «Mythe d'une lingua franca et économie des langues» en el coloquio internacional Communication et mondialisation. Les limites du toutanglais, CNRS, París, 14/11/2012. 
lo expresa Oltermann (2013) recogiendo las palabras del escritor y traductor del Consejo de Europa, Diego Marani:

«Imponer el inglés como lengua oficial de Europa sería profundamente antidemocrático», dice Diego Marani, novelista y responsable de políticas en la Dirección General de Interpretación de la Comisión Europea. No solo no contribuiría a la integración de Europa, sino que podría hacer que el proyecto fuera aún más elitista. El coste total de la labor lingüística en la UE, destaca Marani, equivale más o menos al coste de dos cafés por persona y año: un precio muy barato a cambio de tener más democracia.

La apuesta por el unilingüismo no solo no es rentable económicamente hablando, sino que es nociva desde el punto de vista de la diversidad ya que acarrea un empobrecimiento tanto lingüístico como cultural y, por ende, un empobrecimiento de la ciencia y de la investigación en sentido amplio, y en última instancia (o primera) un empobrecimiento económico y político.

Las últimas cifras del Eurobarómetro indican que sobre la totalidad de la población europea solo el $7 \%$ de la población declara que tiene un muy buen nivel de inglés como lengua extranjera, $17 \%$ un buen nivel y el $11 \%$ solo un nivel básico. Con estas cifras, la imposición de una lengua única excluiría a más del $65 \%$ de los ciudadanos europeos.

La economía de las lenguas no debe reducirse únicamente a la dimensión lingüística, hay que tener en cuenta también la dimensión cultural y científica y valorar los beneficios que supone la divulgación del saber en lengua propia, sea minoritaria o no. Raymond Renard (2006) insiste en la importancia de la protección de la diversidad lingüística, porque en ella reside la autenticidad de la interpretación del mundo a través de la lengua propia, sin filtro. A través de la lengua propia los individuos ven el mundo y lo interpretan a su manera, asegurando así el acceso a lo universal porque todas las lenguas participan en la interpretación del universo puesto que cada cultura produce significados de valor universal (Renard, 2006).

En este sentido, hay que tener en cuenta igualmente que la utilización de la lengua propia para la difusión del saber no hace sino aportar luz y ventajas al acercamiento de los ciudadanos a la ciencia y la cultura. La divulgación del conocimiento requiere de estrategias de didacticidad, fórmulas que permitan el acceso a saberes especializados de un receptor amplio y heterogéneo. Algunas de estas estrategias están relacionadas con mecanismos idiomáticos propios de cada lengua como la fraseología, las expresiones metafóricas, las colocaciones léxicas, etc., que se perderían por el camino si se utiliza una lengua única, común, diferente de la lengua propia del autor. Es cierto que el estándar (vin- 
culado con el inglés) garantiza la transmisión aséptica, científica, del saber, pero no contribuye al potencial divulgativo, didáctico de los conocimientos especializados.

El ciberespacio, en tanto que entorno mediador para la divulgación del saber, plantea nuevos retos y desafíos en pro del plurilingüismo y de la comunicación que dibuja un nuevo paradigma en el que información, comunicación e interacción conviven en un mismo tiempo y espacio.

\section{Retos y desafíos del ciberespacio multilingüe}

El siglo XXI ha despertado en la era de la Información y de la Comunicación. Comunicar hoy en día, en la era de la globalización, es equivalente a negociar, y la negociación (en cualquier ámbito) implica tener en cuenta, la interdisciplinaridad, la diversidad (de locutores, de naturalezas, de realidades, de sociedades, de lenguas, de culturas, etc.).

Para Vizer $(2007,2009)$ «las TIC ya no pueden ser pensadas como meras mediaciones (en el sentido atribuido a los medios de comunicación de masas). Las TIC efectivamente construyen y constituyen nuevas formas, espacios y tiempos de relación social, nuevas formas institucionales, nuevas categorías de aprehensión de la experiencia personal y social, nuevas dimensiones de la cultura». El ciberespacio ha proporcionado, a través de la tecnología 2.0, la posibilidad de desarrollar innumerables tecnologías sociales (blogs, wikis, redes sociales) en las que los usuarios, más o menos anónimos toman la palabra para explicar, debatir, difundir y, en definitiva, construir conocimiento. Según Javier Cremades (2007) «la voz del ciudadano ha encontrado en las nuevas tecnologías canales para participar activamente en la configuración de la opinión pública. Los consumidores, los accionistas, los estudiantes, los ciudadanos corrientes, en definitiva, son ahora más relevantes por que se han convertido en nodos de unas redes sociales cada vez con mayor influencia». Se trata de un fenómeno social denominado «micropoder» por Cremades.

Este micropoder ciudadano en la comunicación ha sido posible gracias a las propias características del tipo de textos que estas tecnologías sociales tecnologías vehiculan:

- Multimodalidad: con la tecnología digital toda la información puede transmitirse a través del mismo canal, lo que provoca la convergencia de varios formatos (audio, vídeo, texto e imagen): «la digitalización ha permitido la integración de los medios de comunicación y de informa- 
ción en las nuevas tecnologías, pasando de la información a la verdadera comunicación interactiva» (Cremades, 2007: 15).

- Reticularidad: Lévy (2007: 49) indica que la estructura reticular que presentan las TIC condiciona la forma comunicativa y la reubicación de los participantes en la comunicación, por lo que construye espacios de interlocución entre usuarios y forma comunidades virtuales de interlocutores.

- Deslocalización geográfica: Castells (2005: 455) indica que se produce «una nueva lógica espacial, el espacio de los flujos -en contraposición con la lógica de los lugares -que permite operar en una red global de interacción que transciende las fronteras nacionales y geográficas», como veremos en el apartado 4.

Volviendo a los datos del Eurobarómetro sobre las lenguas de 2012, se observa que los resultados relacionados con el uso las tecnologías son más optimistas. Estos indican que las lenguas extranjeras son consideradas como una herramienta muy útil para tener acceso a Internet y a otros media. Alrededor de un tercio de los europeos utiliza su primera lengua extranjera para ver películas, la televisión o escuchar la radio (34\%) navegar por Internet (34 \%) y comunicar con los amigos (31\%). Se observa también un alza en la utilización de una segunda lengua en Internet con un aumento de más de siete puntos.

Un cuarto de los encuestados utiliza su primera lengua extranjera en conversaciones de trabajo (25\%) y para leer libros, periódicos o revistas (24\%).

El $17 \%$ utiliza su primera lengua extranjera para escribir correos electrónicos, para leer en el trabajo (16\%) y otro $16 \%$ lo utilizan para comunicar con familiares.

En los primeros años de la década de los noventa el inglés dominaba por completo el ciberespacio (no olvidemos que la Internet que conocemos ahora tiene su origen en estrategias militares de comunicación desarrolladas durante el periodo de la guerra fría por los Estados Unidos). Es en los primeros años noventa cuando este sistema de comunicación traspasa las barreras militares y empieza a introducirse en otros ámbitos, primero comerciales, luego educativos, sociales, etc.

En los últimos diez años la presencia de otras lenguas ha multiplicado hasta por miles el porcentaje, tanto por la creación de contenidos en lengua propia como por la traducción de los mismos a otras lenguas.

Según Oustinoff (2012), en la década comprendida entre el año 2000 y 2010 , la presencia del inglés en la red solo ha aumentado un $281,2 \%$. Decimos «solo» porque a pesar de que se trata de un aumento importante, comparado 
con la progresión de otras lenguas, esta cifra se minimiza. En efecto, el chino, por ejemplo, aparece como la segunda lengua más representada con una participación del 22,6 \% y una tasa de progresión del 1277,4 \%. Pero la progresión más importante, a pesar de que en porcentaje de participación solo representa el 3,3\%, es la del árabe, cuyo aumento ha sido del 2501,5\%, colocándole en séptima posición, detrás del español (tercera posición), japonés (cuarta), portugués (quinta) y alemán (sexta). El francés ocupa la octava posición, el ruso la novena y el coreano la décima. Estas diez lenguas representan el $82 \%$ del total, mientras que el $18 \%$ restante lo ocupan el resto de lenguas (de las 6000 habladas en el planeta).

Ante estos datos, que estamos asistiendo a una rebabelización del mundo, e Internet no hace más que confirmar esta tendencia. Según Oustinoff, parafraseando la expresión de Umberto Eco, para quien la lengua de Europa es la traducción, que la lingua franca de Internet es el plurilingüismo.

Todo ello es reflejo de un fenómeno social y económico: el multilingüismo no se está imponiendo a nivel mundial de manera creciente porque el ciberespacio sea multilingüe, sino porque este es un nuevo espacio comercial, necesario en la economía globalizada, que requiere transacciones multilingües.

En un mundo cada vez más multipolar, donde la globalización está acompañada de las tecnologías de la información y de la comunicación, dominar únicamente la lingua franca es una desventaja, pues implica estar desinformado, subinformado o infrainformado, es decir, el ciudadano que solo utilice esa lingua franca estará en inferioridad de condiciones respecto a quienes dominan varias lenguas. Es lo que Louis-Jean Calvet (2007) ha denominado «la paradoja de la lengua dominante»:

En la era de la globalización estar subinformado o infrainformado es un lujo que no podemos permitirnos, por lo que el dominio de la lingua franca es a todas luces insuficiente.

\section{Iniciativas multilingües en el ciberespacio}

Como hemos visto, el espacio de más rápido crecimiento en comunicación es el ciberespacio. En su entorno estamos asistiendo al final de la hegemonía lingüística, y muestra de ello, además, son algunas iniciativas, institucionales o particulares, que se están llevando a la práctica actualmente en la red que pretenden dar cuenta y preservar la diversidad lingüística y cultural del planeta acercando las lenguas, los pueblos y las culturas al resto del mundo a través de la traducción multilingüe u ofreciendo espacios de expresión a lenguas 
minoritarias, de manera que la información, la ciencia, el conocimiento y la investigación puedan llegar a un público lo más amplio posible. Presentamos a continuación algunas de ellas.

\subsection{Maaya - Red Global para la Diversidad Lingüística ${ }^{7}$}

Se trata de una red creada con la finalidad de contribuir a la revalorización y a la promoción de la diversidad lingüística en el mundo como fundamento de la unidad de la comunicación humana. Creada por iniciativa de la Academia Africana de las Lenguas (ACALM) bajo los auspicios de la Unión Africana, sirve de plataforma de intercambio y de mutualización de saberes compartidos.

La red Maaya surge en 2005 en el marco de la Cumbre Mundial sobre la Sociedad de la Información (CMSI) en la cual la diversidad cultural y lingüística en el ciberespacio fue identificada como una de las prioridades. A pesar de haber nacido en el contexto de dicha cumbre, el campo de acción de esta red no se limita al ciberespacio. Maaya es una red multilateral que implica a diferentes actores: sociedad civil, gobiernos y organizaciones internacionales.

Entre sus objetivos se encuentran:

- Incitar a los gobiernos e instituciones a adoptar y aplicar medidas que favorezcan un multilingüismo equitativo.

- Fomentar la educación bi- o multilingüe basada en la lengua materna, en todos los niveles de la educación y en todas partes del mundo, con el fin de garantizar una igualdad social y entre los sexos.

- Promover la localización de los programas informáticos y el acceso igualitario de todas las lenguas al ciberespacio.

- Facilitar el empoderamiento de las comunidades lingüísticas de todo el mundo de manera que tengan el control del desarrollo de su propia lengua y que puedan utilizarla y defenderla.

- Contribuir a la creación y al intercambio de recursos lingüísticos.

- Dar seguimiento a la implementación de políticas lingüísticas y constituir un centro de orientación para proyectos de investigación lingüística.

7. Red Maaya (Red Mundial para la Diversidad Lingüística): http://www.maayajo.org 
Cabe destacar que el sitio web de Maaya se presenta en cuatro lenguas: español, francés, portugués e inglés, y que desde 2010 celebra regularmente un simposio internacional sobre multilingüismo y ciberespacio.

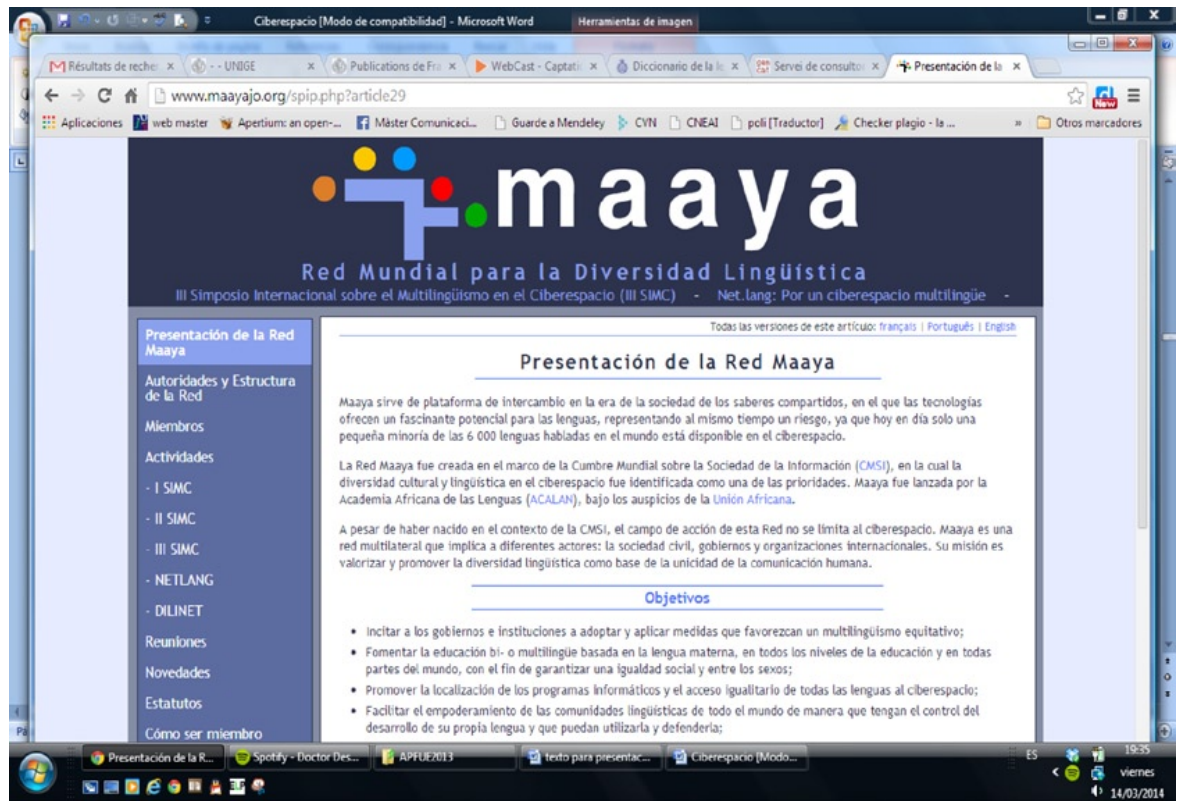

Imagen 1: Red Maaya: http://www.maayajo.org

\subsection{Linguamón - Casa de las Lenguas ${ }^{8}$}

Otra iniciativa que trabaja en la misma línea es Linguamón - Casa de las Lenguas. Se trata de un organismo gubernamental creado en 2005 e integrado por la Generalitat de Cataluña y el Ayuntamiento de Barcelona. Su misión es acercar el mundo de las lenguas a los ciudadanos, hacer que la sociedad viva la riqueza lingüística de manera positiva, crear conciencia por la sostenibilidad de la diversidad lingüística y difundir las grandes posibilidades que ofrecen las lenguas y sus comunidades.

Por otra parte, mediante servicios especializados, llevan a cabo iniciativas en torno a las lenguas para contribuir a la mejora del desarrollo social y económico de la sociedad, de manera que los modelos de gestión del multilingüismo pro-

8. Linguamón - Casa de las Lenguas: http://www10.gencat.cat/casa_llengues/AppJava/es/index.jsp 
puestos incidan positivamente en la cohesión social, la competitividad y la economía de un país, así como en el desarrollo social y laboral de la ciudadanía.

$\mathrm{Su}$ labor se centra en:

- Favorecer la sostenibilidad lingüística promocionando la preservación, uso, reconocimiento y aprendizaje de las lenguas en el mundo de manera que el progreso social y económico no las ponga en peligro.

- Favorecer la presencia del multilingüismo en el ciberespacio facilitando comunicaciones multilingües.

- Gestionar el multilingüismo proyectando internacionalmente modelos de éxito que faciliten la convivencia de lenguas en un mismo territorio o entre un mismo grupo de personas.

Linguamón-Casa de las lenguas presenta su sitio web en nueve lenguas: las cuatro lenguas oficiales del Estado español -catalán, español, eusquera y gallego-, inglés, francés como lenguas internacionales y una lengua inclusiva poco usual en la red como es la lengua de signos y otras dos igualmente poco habituales, el occitano y una variante del bereber, el tamazight.

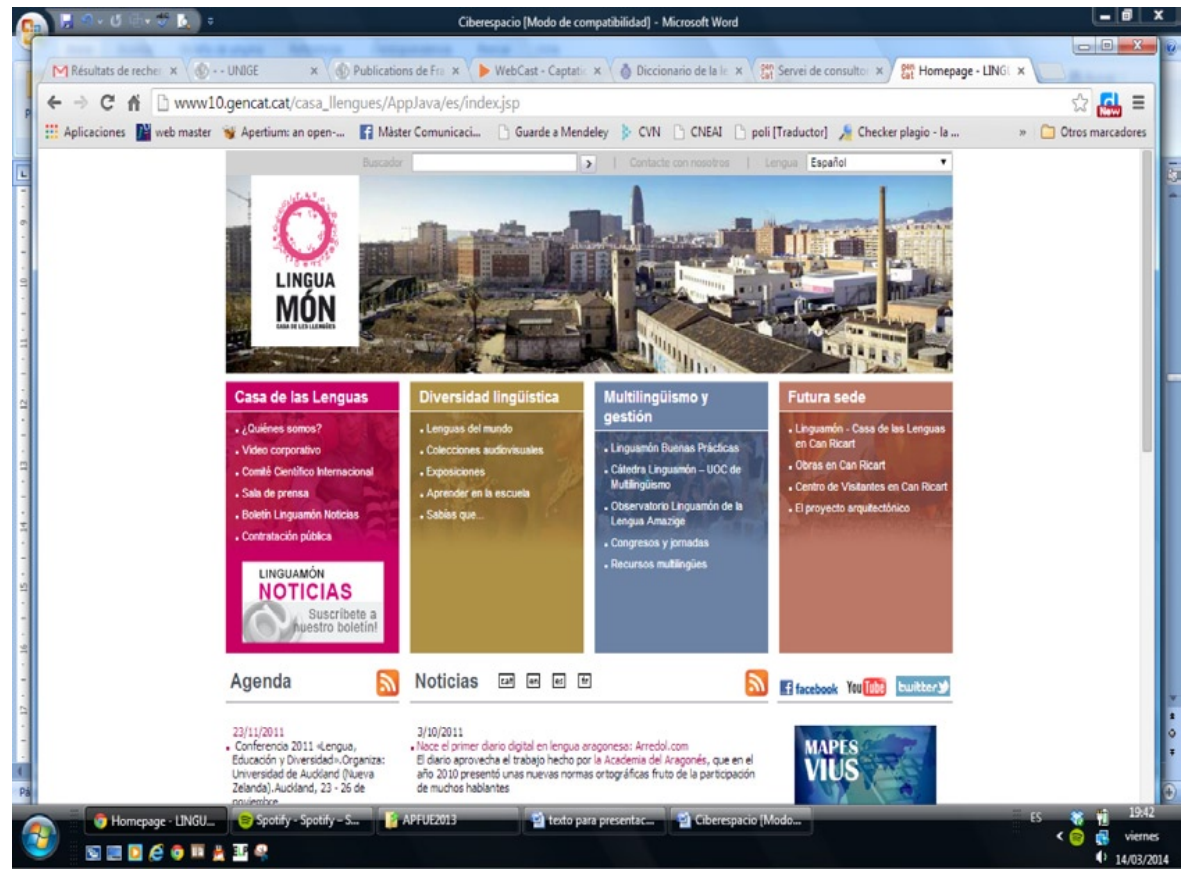

Imagen 2: Linguamón-La Casa de las Lenguas: www.linguamon.cat 


\subsection{Global Voices: el mundo habla ¿estás escuchando?}

La frase que acompaña al nombre de esta red es muy significativa. Es una red internacional de más de 800 blogueros y traductores de todo el mundo que trabajan para reportar informes de blogs y medios ciudadanos de todas partes, con énfasis en las voces que no son escuchadas habitualmente en los principales medios internacionales. Se plantea como reacción a la proliferación de medios de comunicación internacionales en lengua inglesa que ignoran muchos asuntos que son importantes para gran número de ciudadanos del mundo que no dominan esta lengua, Global Voices pretende corregir algunas de esas desigualdades provocadas por los medios, aprovechando justamente el poder de los medios de comunicación de los ciudadanos.

Global Voices presenta dos variantes del sitio: Global Voices Advocacy y Rising Voices.

Global Voices Advocacy trabaja en red para proteger la libertad de expresión y el libre acceso a la información en línea. Informan sobre las amenazas a la expresión en línea, comparten recursos para la defensa de las obras y las palabras de los internautas y trabajan para mejorar la política de uso y práctica de Internet en el mundo.

Rising Voices, por su parte, es otra iniciativa de divulgación que ayuda a comunidades marginadas a difundir sus proyectos y encontrar recursos y financiación para llevarlos a cabo a través de la microsubvenciones principalmente.

Todos ellos abogan por la libertad de expresión en todo el mundo y protegen los derechos de los periodistas ciudadanos para informar sobre eventos y opiniones sin temor a la censura o la persecución. Global Voices presenta contenidos traducidos a más de 35 idiomas por traductores voluntarios que han formado el proyecto Lingua.

Global Voices busca agrupar, conservar y amplificar la conversación global en línea arrojando luz a los lugares y gente que otros medios de comunicación a menudo ignoran. Trabajan para desarrollar herramientas, instituciones y relaciones que ayuden a todas las voces, en todas partes, a ser oídas. De esta manera reducen la brecha informacional que sufren muchos ciudadanos por el hecho de no conocer la lengua dominante, facilitando así el acceso a la información y al conocimiento. Su ámbito de acción se extiende a más de 167 países. 


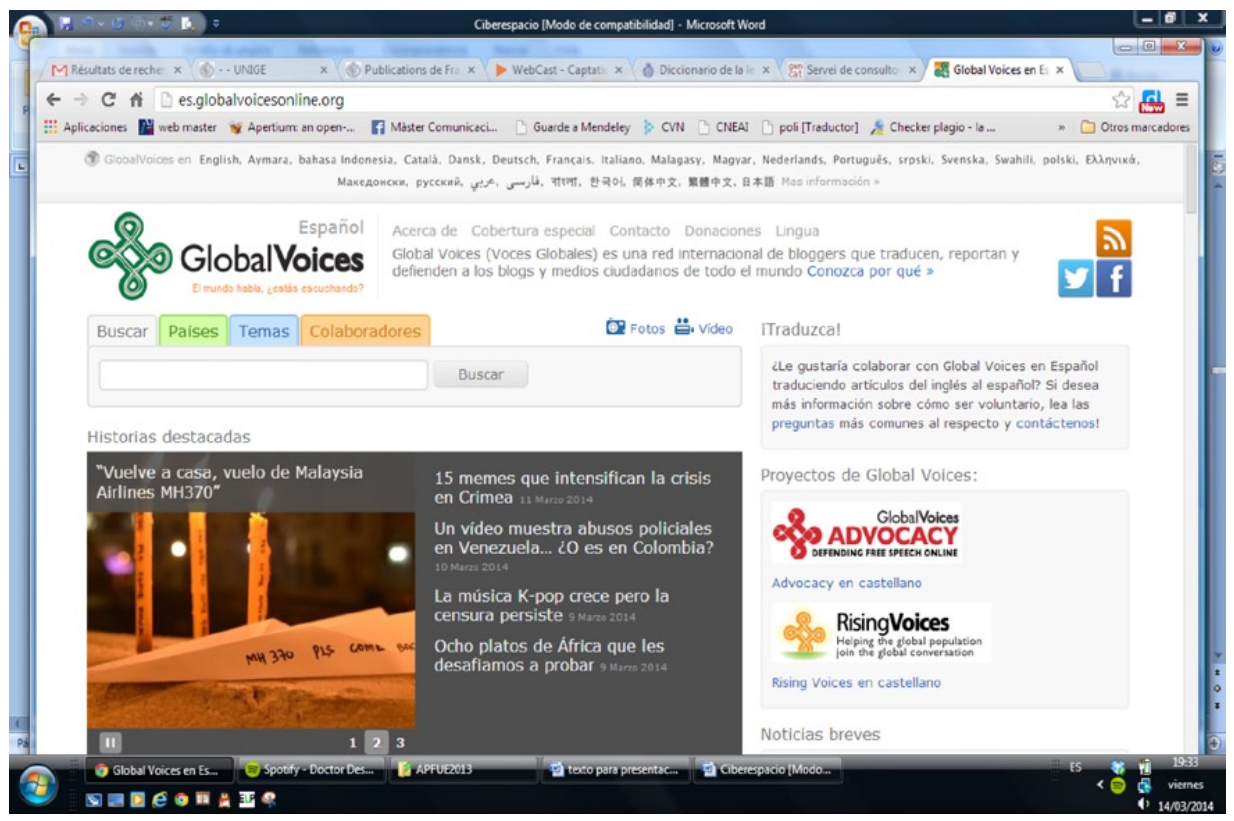

Imagen 3: Global Voices: http://es.globalvoicesonline.org

\subsection{WorldWideScience.org ${ }^{9}$}

WorldWideScience.org es una plataforma multilingüe que se presenta como una puerta abierta a la ciencia mundial facilitando la difusión del conocimiento científico y de los resultados de investigación que se llevan a cabo en cualquier rincón del planeta. A través de esta plataforma se pueden hacer búsquedas en tiempo real en 70 bases de datos científicas procedentes de 66 países en lenguas diversas. Todo está ligado a un buscador multilingüe y un programa de traducción automática en diez lenguas, de momento: alemán, árabe, chino, coreano, español, francés, inglés, japonés, portugués y ruso.

William F. Brinkman, director de la Oficina de Ciencia, del Departamento de Energía de EE. UU., promotor de esta plataforma explica:

En un mundo cada vez más interconectado, la solución de los desafíos globales de la ciencia requiere una rápida comunicación del conocimiento científi-

9. WorldWideScience.org: http://worldwidescience.org/ 
co. Romper la barrera del idioma a través de WorldWideScience.org ayudará a erosionar las fronteras y construir redes de investigación a través del DOE, ${ }^{10}$ la nación y alrededor del mundo.

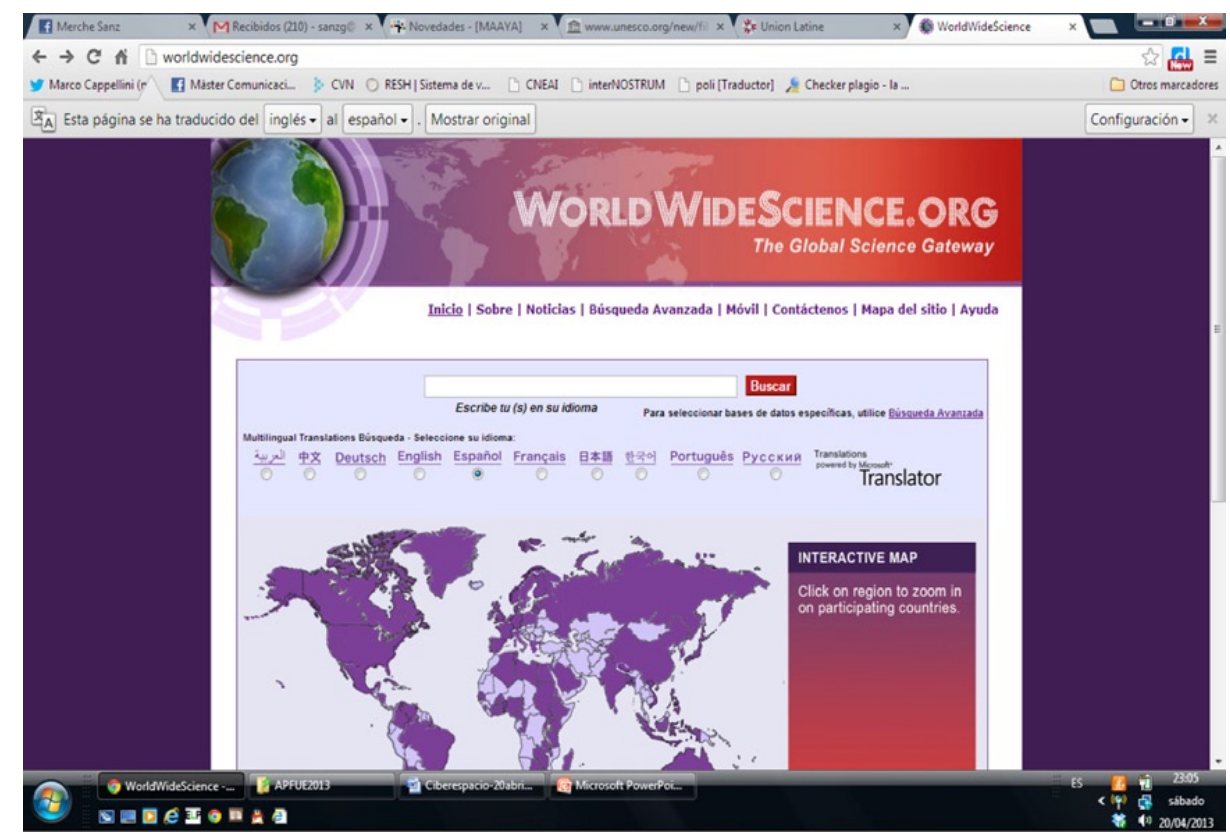

Imagen 4: WorldWideSience.org

\subsection{Société Européenne des auteurs: Borges/TLhub}

El Proyecto Borges/Tlhub (Traducción Literaria y Hub) de la Sociedad Europea de Autores es una plataforma de Internet dedicada a la traducción colaborativa de obras en las diferentes lenguas habladas en Europa en la que participan tanto autores como traductores o editores.

El Proyecto Borges se está desarrollando en la actualidad. Una primera versión se lanzó a finales de 2011. La idea es desarrollar una herramienta de Internet utilizando la tecnología web 2.0, una herramienta abierta a disposición de los escritores, traductores y editores, para facilitar las relaciones entre las diferentes lenguas.

10. U.S. Department of Energy's Office. 
Borges/TLHUB se concibe como una comunidad con acceso semiabierto al que solo los autores, traductores y editores tienen acceso completo a los contenidos. Los «lectores» también podrán tener acceso, pero con algunas restricciones.

TLHUB es una herramienta de traducción colaborativa, sin ánimo de lucro y de cooperación basada en la tecnología de código abierto, que se dedica a construir una comunidad más allá de la traducción de idiomas y naciones. Tiene la intención de crear un servicio público universal para los traductores y autores. TLHUB respeta los derechos de autor.

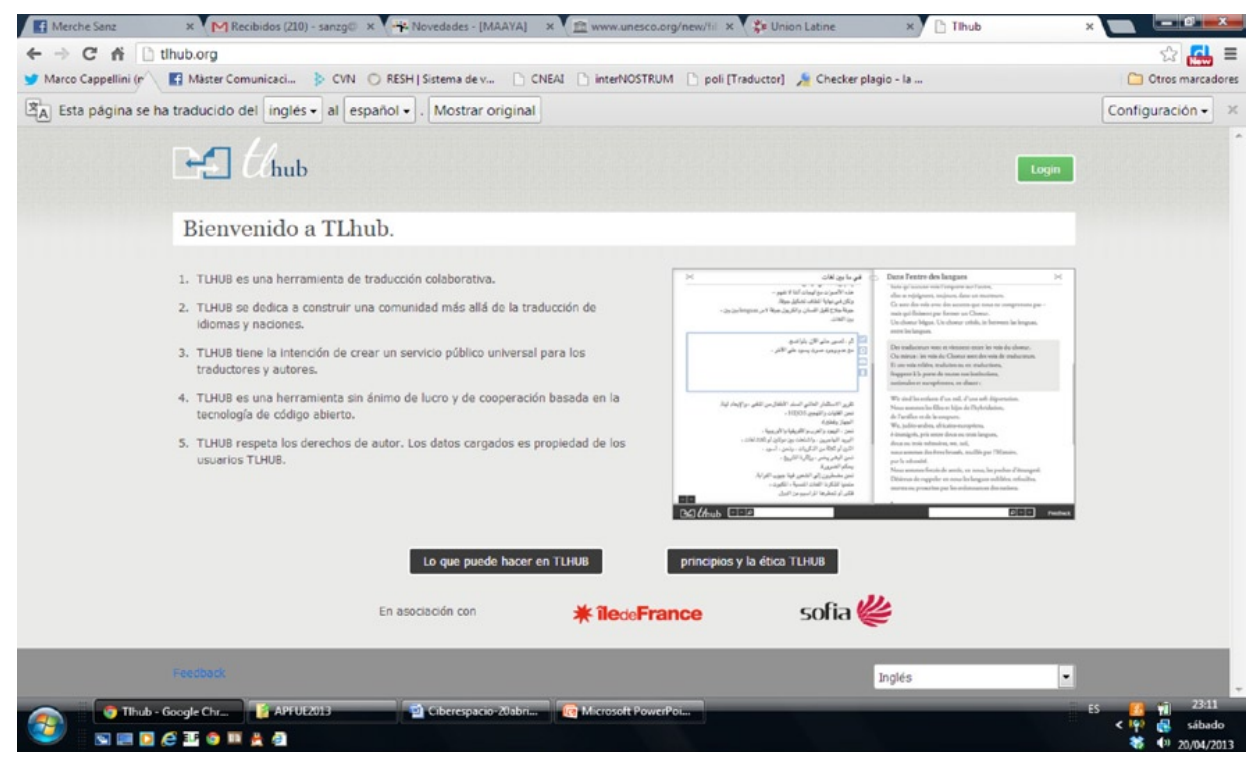

Imagen 5: Proyecto Borges/TlHuB

\subsection{Wikipedia}

Si pensamos en la colaboración, en la mutualización de esfuerzos y difusión del conocimiento, no podemos dejar de mencionar el proyecto Wikipedia, enciclopedia libre editada de manera colaborativa y conocida a nivel mundial, que actualmente publica contenidos en 287 lenguas. ${ }^{11}$ Se trata del más claro

11. Véase http://es.wikipedia.org/wiki/Anexo:Wikipedias 
ejemplo de entorno global en el que cada persona tiene acceso libre y gratuito al conjunto del conocimiento humano, tal y como lo explica uno de sus cofundadores, Jimmy Wales: ${ }^{12}$

Tengo la intención de obtener una copia de Wikipedia para cada una de las personas en el planeta en su propio idioma. [...] Imaginemos un mundo en que cada persona tiene el acceso libre y gratuito a la suma de todo el conocimiento humano. Es lo que estamos haciendo.

Con el proyecto Wikipedia pretende «crear y distribuir una enciclopedia libre, de la más alta calidad posible, a cada persona del planeta, en su idioma», para conseguir «un mundo en el que cada persona del planeta tenga acceso libre a la suma de todo el saber de la humanidad».

\subsection{Redes sociales de comunicación}

Y finalmente otro fenómeno cada vez más en expansión, que está contribuyendo a la presencia de múltiples lenguas en el ciberespacio, son las redes sociales, tanto por los contenidos que en ellas se publican como por las lenguas de interfaz que sostienen estas plataformas. Por solo citar algunas, diremos que la interfaz de Twitter se presenta en 37 lenguas, o Facebook en 104, Youtube en 61, Skype en 38, Hi5 en 24, Linkedin en 22, Second Life en 9, Tuenti en 7, Picasa en 40, Flickr en 10, Google + en 61, Blogger en 62, etc.

Algunas de estas redes, como Twitter, cuentan con centro de traducciones formados por una comunidad de traductores voluntarios que lo enriquecen incorporando continuamente nuevas lenguas de interfaz; sin embargo, para la mayor parte de ellas la principal base de esta traducción son los traductores automáticos, con las deficiencias que ello conlleva. Conscientes de este problema, todas estas plataformas incorporan la posibilidad de mejorar la traducción colaborando así con el proceso de creación y de edición, y sobre todo evidencian la inquietud cada vez más creciente de romper con la hegemonía lingüística presente en la red hasta hace una década.

12. Entrevista realizada en 2004 para el sitio Slashdot: News for nerds, stuff that matters: http:// slashdot.org/story/04/07/28/1351230/wikipedia-founder-jimmy-wales-responds 


\section{Conclusión}

Los derechos manifestados por la declaración universal de la UNESCO sobre la diversidad cultural deberían entenderse como la base sobre la que se asiente la actuación de cualquier política. Preservar y promover la diversidad y el pluralismo culturales y lingüísticos como medios para la difusión y divulgación de la ciencia y del conocimiento resulta una necesidad imperiosa en cualquier ámbito.

Para ello contamos con dos bazas importantes y fundamentales: el ciberespacio, por un lado, que tal y como hemos mostrado en este viaje por las iniciativas plurilingües, se manifiesta cada vez más como un entorno teñido y plagado de color cultural y lingüístico, recogiendo la imagen de la cita con la que abríamos nuestro artículo, y por otro lado, las generaciones que hoy se encuentran en edad escolar, los nativos digitales, que entienden el ciberespacio como un entorno natural de comunicación y de aprendizaje y que en definitiva, serán quienes tengan que defender estos derechos fundamentales, como mencionábamos en los primeros párrafos de este artículo.

\section{Referencias bibliográficas}

Calvet, L. J. (2007): «La mondialisation au filtre des traductions», París, Hermès, 49: 47-57.

Castells, M. (2005): La sociedad red, Madrid, Alianza.

Colloque internationnal Communication et mondialisation. Les limites du tout-anglais, CNRS, París, 14/11/2012, <http://webcast.in2p3.fr/eventscommunication_et_mondialisation> [16/03/2014].

COMISIÓN EUROPEA (2012): EUROBAROMÈTRE spécial 386 «Les européens et leurs langues», <http://ec.europa.eu/public_opinion/archives/ebs/ebs_386_ fr.pdf $>$ [16/03/2014].

Cremades, J. (2007): Micropoder. La fuerza del ciudadano en la era digital, Madrid, Espasa-Calpe.

Fondevila Silva, P.; J. J. Sánchez Baena (2004-2007): «Un nexo en la historia de la comunicación naval: la lengua franca mediterránea», Contraste. Revista de Historia Moderna, 13: 157-182.

Grin, F. (2012): «Mythe d'une lingua franca et économie des langues». Communication et mondialisation. Les limites du tout-anglais, CNRs, París, 14/11/2012, <http://webcast.in2p3.fr/events-communication_et_mondialisation> [16/03/2014]. 
LÉvy, P. (2007): Cibercultura, Barcelona, Anthropos.

Massééou, A. (2012): «L'avenir se parle, s'écrit et se lit dans toutes les langues», NET.LANG. Réussir dans le cyberespace multilingue, Caen, $\mathrm{C} \& \mathrm{~F}$ Éditions.

Oltermann, Ph. (2013): «Something in common: should English be the official language of the Eu?», The Guardian, <http://www.theguardian.com/ world/2013/apr/24/europa-english-official-language-eu> (24/04/2013), [16/03/2014].

OustinofF, M. (2009): «Plurilinguisme et traduction à l'heure de la mondialisation», Conferencia realizada en Expolangues 2009. Ed. Clé des Langues, $<$ http://cle.ens-lyon.fr/servlet/com.univ.collaboratif.utils.lecturefichiergw?id fichier $=1332154733523>[16 / 03 / 2014]$.

- (2012): «L'anglais ne sera pas la lingua franca d'Internet» en VANNINI, L.; H. Le Crosnier (eds.) (2012): net.lanG. Réussir dans le cyberespace multilingue, Caen, C\&F Éditions.

- (2013): «La diversité linguistique, enjeu central de la mondialisation», Revue Française des Sciences de l'Information et de la Communication, 2, $<\mathrm{http} / / / \mathrm{rfsic}$.revues.org/328?lang=en $>$ [16/03/2014].

RENaRD, R. (2006): Une éthique pour la francophonie. Questions de politique linguistique, París, Didier Érudition, Édition du CIPA.

UnesCo (1948): Declaración Universal de Derechos Humanos, <http://portal. unesco.org/es/ev.php-URL_ID $=26053 \& \mathrm{URL}_{-}$DO=DO_TOPIC\&URL_ SECTION $=201 . \mathrm{html}>[16 / 03 / 2014]$.

- (2001): Declaración universal de la UNESCO sobre la diversidad cultural. $<$ http://unesdoc.unesco.org/images/0012/001271/127160m.pdf $>$ [16/03/2014].

Vannini, L.; H. Le Crosnier (eds.) (2012): net.lang. Réussir dans le cyberespace multilingue, Caen, $\mathrm{C} \& \mathrm{~F}$ Éditions.

Vizer, E. A. (2007): «Hacia una ecología social y estratégica de la comunicación». Ponencia del Colóquio Transfronteiras. Publ. Secret. de Ciencia y Téc., Facult. de C. Sociales, Universidad de Buenos Aires.

- (2009): «Etapas de la cultura tecnológica y creación de valor» en FAGUNDES Hausen, D.; V. Cruz Brittos (eds.): Economía Política, Comunicaçao e cultura, Porto Alegre, Brasil, Pontificia Universidade Católica do RGS: 181-201. 